\title{
Effect of surgical work volume on postoperative complication: superiority of specialized center in gastric cancer treatment
}

\author{
Birendra Kumar Sah • Zheng Gang Zhu • \\ Ming Min Chen • Ming Xiang • Jun Chen • Min Yan • \\ Yan Zhen Lin
}

Received: 21 March 2008 / Accepted: 27 May 2008 /Published online: 27 June 2008

(C) The Author(s) 2008

\begin{abstract}
Purpose We investigated the risk factors for early postoperative complications after gastric cancer surgery.

Materials and methods The data from a total of 273 patients with gastric cancer were analyzed by univariate and multivariate analysis. We applied physiological and operative severity score for the enumeration of morbidity and mortality (POSSUM) to compare risk-adjusted surgical outcomes among different surgical units.

Results Among the preoperative variables, patient gender, chronic obstructive pulmonary disease, surgical unit, and intraoperative blood loss were independent risk factors for a higher rate of postoperative complications. There were significant differences in complication rates among different surgical units $(P=0.001)$. The observed-to-expected morbidity ratio (O-to-E ratio) ranged from 0.81 to 1.63 . Units with low surgical work volume had higher complication rates. Postoperative length of stay was significantly shorter $(P=0.000)$ and the rate of moderate and severe complications was significantly lower $(P=0.001)$ in specialized unit.
\end{abstract}

B. K. Sah • Z. G. Zhu $(\bowtie) \cdot$ M. M. Chen • M. Xiang • J. Chen • M. Yan • Y. Z. Lin

Department of General Surgery, Rui Jin Hospital,

197 Rui Jin Er Road,

Shanghai 200025, China

e-mail: rjzhuzhenggang@hotmail.com

B. K. Sah • Z. G. Zhu • M. M. Chen - M. Xiang - J. Chen •

M. Yan· Y. Z. Lin

School of Medicine, Shanghai Jiao Tong University,

Shanghai, China

B. K. Sah • Z. G. Zhu • M. M. Chen • M. Xiang • J. Chen •

M. Yan $\cdot$ Y. Z. Lin

Shanghai Institute of Digestive Surgery,

Shanghai, China
Conclusions POSSUM is a valid system for risk-adjusted evaluation of surgical outcomes. We conclude that surgical experience and work volume greatly influence the outcome, with overall surgical outcome in specialized centers superior to that in other units. Hence, gastric cancer surgery should be performed in specialized centers. Risk factors identified in this study need further confirmation by a prospective study involving a larger cohort.

Keywords Surgical work volume · Postoperative complications · Gastric cancer · POSSUM

\section{Introduction}

The practice of gastric cancer surgery is highly heterogeneous among different units, and there are obvious variations in postoperative complication rates according to the extent of surgery, especially in the hands of inexperienced surgeons. The optimal extent of surgery for gastric cancer is highly controversial, and postoperative complications after gastrectomy are still a major challenge in surgical practice; therefore, further research to minimize the extent of surgery but still obtain an optimal result is needed. The rate of early postoperative complications is as important as the rate of survival and should be seriously considered in order to optimize the extent of surgery. Furthermore, the outcome of surgical intervention is not solely dependent on the abilities of the surgeon. The patient's physiological status, the disease that requires surgical correction, the nature of the operation, and the preoperative and postoperative support services have a major effect on the ultimate outcome [1]. Simply collecting outcome data alone is not sufficient to reflect treatment quality because to compare postoperative complication data 
directly, the original populations must be identical. Surgeons treating a predominantly young population would be expected to achieve lower complication rates than others serving areas with an elderly population [2]. The unit that selects only low-risk cases achieves a low complication rate and therefore attracts more patients, whereas the unit that cannot select only low-risk cases is left with a worsening case mix [1]. Monitoring crude death rates can mask the effects of case mix; surgeons who work in impoverished inner-city hospitals or tertiary referral centers may feel disadvantaged compared with their colleagues who elect to treat fit patients or work in an affluent area [3].

Comparison of surgical outcome is made even more difficult by vague explanations of complications and lack of standard auditing methods. Physiological and operative severity score for the enumeration of morbidity and mortality (POSSUM) has been proposed as a method for standardizing patient data so that direct comparisons of patient outcomes can be made [2]. There are many scoring systems; but, to date, POSSUM is the most promising for auditing surgery, and it has been extensively used in various surgical specialties $[3,4]$. It is a reliable instrument for comparing internal audits of the same unit during two different periods [5]. When used correctly, POSSUM can effectively compare outcomes between surgeons and between hospitals. The POSSUM system allows a numerical prediction of mortality and morbidity for an individual patient, and it enables comparative audits with a method for adjustment of case mix. Comparison can be made over time and can be easily adjusted to radical changes in health care in the future $[1,6,7]$. In addition, POSSUM has definitions for postoperative complications, which can be used as standard terms while comparing several surgical units.

Here, we investigated the risk factors for early postoperative complications after gastric cancer surgery and we applied POSSUM to compare risk-adjusted surgical outcomes among different units of a single hospital

\section{Materials and methods}

Characteristics of patients

A total of 273 patients who underwent operations for gastric cancer during 2006 were included in the study (Table 1). The median age of the patients was 59 years (range 22-89 years). The ratio of the incidence of gastric cancer in male and female patients was approximately 7:3. The data were collected directly by comprehensive review of the original records of all patients. Six patients with missing data required for the POSSUM calculations were excluded. However, there were no deaths or major complications in the excluded cases according to information provided by the hospital database.

\section{Definition of different units}

All the operations were performed in five general surgery departments. To obtain uniformity among the patients, operations performed in laparoscopic and thoracic surgery departments were excluded. Three units with fewer than 30 cases were merged together for statistical analysis. Finally, three units (A, B, and C) were formed for comparison of their surgical outcomes. Unit B comprises three general units. Unit $\mathrm{C}$ is a specialized unit for gastric cancer surgery.

\section{Extent of surgery}

The majority of operative surgeons were consultants; very few of them were vice-consultants. All the patients with early and resectable advanced gastric cancer (without

Table 1 Demographic data of the patients

\begin{tabular}{|c|c|c|c|c|}
\hline Units & & A & $\mathrm{B}$ & $\mathrm{C}$ \\
\hline & No of cases & 61 & 55 & 157 \\
\hline & Median (range) age, year & $56(57)$ & $61(56)$ & $58(58)$ \\
\hline Physiological score & Median (range) & $16(12-33)$ & $16(12-38)$ & $15(12-32)$ \\
\hline Operative score & Median (range) & $18(16-29)$ & $18(16-28)$ & $18(16-28)$ \\
\hline \multicolumn{5}{|l|}{ Diagnosis } \\
\hline & Primary gastric cancer & 61 & 53 & 152 \\
\hline & Gastric stump cancer ${ }^{\mathrm{a}}$ & 0 & 2 & 5 \\
\hline \multicolumn{5}{|c|}{ No. of procedures $(\%)$} \\
\hline & Partial gastrectomy & $40(65.6)$ & $40(72.7)$ & $119(75.8)$ \\
\hline & Total gastrectomy & $15(24.6)$ & $13(23.6)$ & $31(19.7)$ \\
\hline & Gastrojejunostomy $^{\mathrm{b}}$ & $6(9.8)$ & $2(3.6)$ & $7(4.5)$ \\
\hline
\end{tabular}

\footnotetext{
${ }^{\mathrm{a}}$ Including recurrent gastric cancer

${ }^{\mathrm{b}}$ Palliative surgery, tumor not resected
} 
significant distant metastases) underwent gastrectomies with D2 lymphadenectomy. Because of inadequate numbers of harvested lymph nodes, we could not document all the pathological data according to TNM classification.

\section{Application of POSSUM system}

POSSUM data were collected on a standard sheet, and morbidity was calculated as described by the original authors [8]. A comprehensive explanatory sheet was prepared to minimize any confusion in defining POSSUM variables.

\section{Definition of morbidity}

The only study endpoint was analysis of inpatients' postoperative complications. Complications were recorded in accordance with the definitions reported by Copeland et al. [8]. However, there were a considerable number of complications that were not covered by their definitions. Therefore, any undefined complication requiring a postoperative stay of longer than 15 days was recorded as innominate in this study. Such complications were only recorded for the overall auditing of all postoperative complications and did not affect the POSSUM calculation.

\section{Statistical analysis}

The statistical analysis was performed with the Statistical Package for Social Science (SPSS) version 13.0 for
Windows (SPSS, Inc, Chicago, IL, USA). Univariate analysis was performed using the chi square test or Fisher's test. Multivariate analysis was performed using the logistic regression model for analysis of risk factors for postoperative complications. A $P$ value of less than 0.05 was considered statistically significant.

The exponential analysis method was used for prediction of morbidity [9]. The significance of the predictive value was assessed by the logistic regression model $\chi^{2}$. The observedto-predicted operative morbidity ratio (O-to-E ratio) for POSSUM was calculated for each unit. An O-to-E ratio less than 1 implies a performance that was better than expected and a ratio greater than 1 indicates a performance that was worse than expected. Crude operative morbidity rates were statistically analyzed by means of $2 \times k$ tables - comparison of several proportions. Fisher's exact test was used to test the significance of differences in duration and severity of postoperative complications among different units.

\section{Results}

Univariate analysis revealed that the preoperative variables, unit $\mathrm{B}$, gender, concomitant chronic obstructive pulmonary disease (COPD), and intraoperative blood loss, were significantly associated with a higher rate of postoperative complications. There were no significant associations with age, anemia, hypoalbuminemia, extent of malignancy, or other variables in POSSUM (Table 2). Similarly, multivariate

Table 2 Univariate analysis of risk factors for early postoperative complications

\begin{tabular}{|c|c|c|c|c|c|}
\hline \multirow[b]{2}{*}{ Variables } & & \multicolumn{2}{|c|}{ Complication } & \multirow[t]{2}{*}{$\%$ complication rate } & \multirow[t]{2}{*}{$P$ value } \\
\hline & & Yes & No & & \\
\hline \multirow[t]{3}{*}{ Units } & A & 22 & 39 & 36.1 & 0.004 \\
\hline & $\mathrm{B}$ & 31 & 24 & 56.4 & \\
\hline & $\mathrm{C}$ & 49 & 108 & 31.2 & \\
\hline \multirow[t]{2}{*}{ Gender } & Male & 61 & 125 & 32.8 & 0.023 \\
\hline & Female & 41 & 46 & 47.1 & \\
\hline \multirow[t]{3}{*}{ Age, years } & $<60$ & 56 & 95 & 37.1 & 0.293 \\
\hline & $61-70$ & 25 & 52 & 32.5 & \\
\hline & $>70$ & 21 & 24 & 46.7 & \\
\hline \multirow[t]{2}{*}{ COPD } & Present & 31 & 30 & 50.8 & 0.014 \\
\hline & Absent & 71 & 141 & 33.5 & \\
\hline \multirow[t]{2}{*}{ Anemia } & Present & 60 & 85 & 41.4 & 0.144 \\
\hline & Absent & 42 & 86 & 32.8 & \\
\hline \multirow[t]{2}{*}{ Hypoalbuminemia } & Present & 9 & 22 & 29.0 & 0.309 \\
\hline & Absent & 93 & 149 & 38.4 & \\
\hline \multirow[t]{2}{*}{ Hyperglycemia } & Present & 9 & 16 & 36.0 & 0.883 \\
\hline & Absent & 93 & 155 & 37.5 & \\
\hline \multirow[t]{2}{*}{ Intraoperative blood loss } & $<500 \mathrm{ml}$ & 46 & 119 & 27.9 & 0.000 \\
\hline & $>500 \mathrm{ml}$ & 56 & 52 & 51.9 & \\
\hline \multirow[t]{2}{*}{ Malignancy } & Primary tumor & 33 & 66 & 33.3 & 0.299 \\
\hline & Metastases & 69 & 105 & 39.7 & \\
\hline
\end{tabular}


Table 3 Multivariate analysis of risk factors for early postoperative complications

\begin{tabular}{lllll}
\hline Variables & & Odd ratio & $95 \%$ CI & $P$ value \\
\hline Gender & Male & 1 & & \\
& Female & 1.884 & $1.074-3.304$ & 0.027 \\
Units & A & 1 & & \\
& B & 3.108 & $1.384-6.981$ & 0.006 \\
\multirow{2}{*}{ COPD } & C & 0.963 & $0.495-1.876$ & 0.913 \\
\multirow{2}{*}{ Blood loss } & Absent & 1 & & \\
& Present & 2.251 & $1.207-4.199$ & 0.011 \\
& $<500 \mathrm{ml}$ & 1 & & \\
& $>500 \mathrm{ml}$ & 2.862 & $1.666-4.916$ & 0.000 \\
\hline
\end{tabular}

analysis identified that the preoperative variables, unit B, gender, COPD, and intraoperative blood loss, were independent risk factors for postoperative complications (Table 3). We further tested for the uniformity of these variables among different units (wards), which revealed that there were no significant differences in gender $(P=0.355)$, COPD $(P=$ $0.499)$, or intraoperative blood loss $(P=0.060)$ among patients in different units. Furthermore, there were no significant differences in physiological score (PS; $P=0.34$ ) and operative severity score (OSS; $P=0.08$ ) among patients among different units.

The overall O-to-E ratio was 1.01 and a chi square test showed a significant lack of fit $\left(\chi^{2}=2.156,4 d f, P=0.707\right)$, i.e., observed values were not significantly different from predicted values. The overall surgical outcome (Fig. 1) was acceptable because the observed value was close to the value predicted by POSSUM. Patients in all units were homologous as there was no significant difference in their PS $(P=0.34)$ and OSS $(P=0.08)$, but there was a significant difference in observed complication rates among the different units $(P=0.001)$. At the same time, there was a significant variation in risk-adjusted morbidity rates (Table 4).

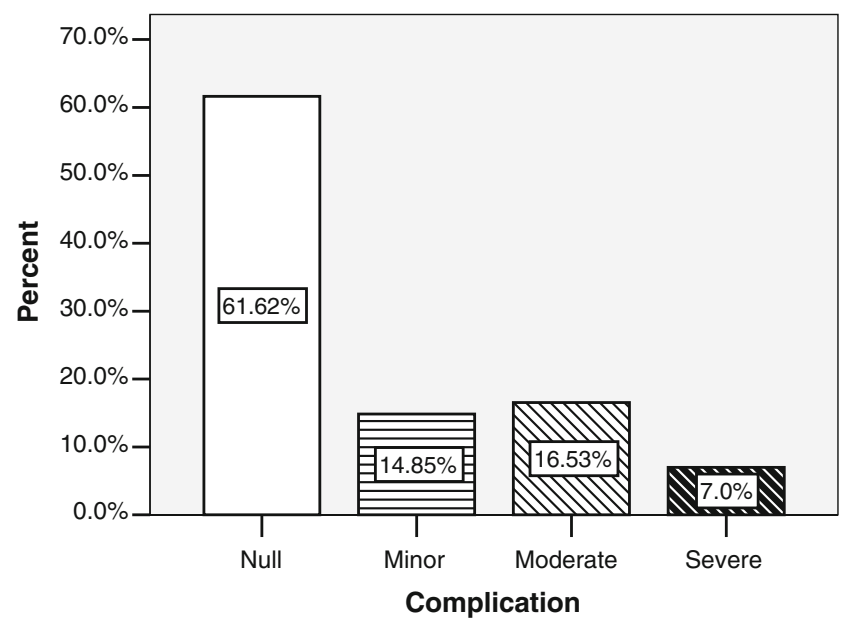

Fig. 1 Distribution chart of overall surgical outcome
Table 4 Risk-adjusted morbidity rates for different wards

\begin{tabular}{lcccc}
\hline Unit & $\begin{array}{l}\text { No. of } \\
\text { patients }\end{array}$ & $\begin{array}{l}\text { Observed } \\
\text { morbidity (\%) }\end{array}$ & $\begin{array}{l}\text { Expected } \\
\text { morbidity (\%) }\end{array}$ & O to E \\
\hline A & 61 & $22(36.1)$ & $26(42.6)$ & 0.85 \\
B & 55 & $31(56.4)$ & $19(34.5)$ & 1.63 \\
C & 157 & $49(31.2)$ & $60(38.2)$ & 0.81 \\
\hline \multicolumn{7}{l}{ O to E Observed to expected }
\end{tabular}

Unit $\mathrm{C}$, which is the specialized center for gastric cancer surgery, had the lowest morbidity rate with null mortality, while conducting the highest number of operations $(57.5 \%)$. The overall surgical outcome was also superior to the other units. Unit B comprises three units having fewer than 30 operations in each; it had the highest morbidity rate, and even the rate of anastomotic leakage was as high as $9 \%$ (Table 5). Details concerning the complications in each ward are shown below in Table 5 . The number of complications is not equal to number of patients because multiple complications were possible in a single patient. Postoperative infection was the most frequent among the different types of complications.

After the calculation of complications using POSSUM, all complications were stratified according to severity (Fig. 1) and postoperative length of stay (Fig. 2). The Rui Jin Hospital (RJH) system for classification of complications (Table 6) was applied for the classification according to severity. Except the "innominate" complication, all the complications in this classification was strictly defined according to the definition of morbidity in POSSUM system. Patients with multiple complications were grouped into the highest level of their respective complications, e.g., a patient

Table 5 Complications per unit

\begin{tabular}{llccc}
\hline \multirow{2}{*}{ Complication } & & \multicolumn{3}{c}{ Frequency (\%) in different units } \\
\cline { 3 - 5 } & & A & B & C \\
\hline Hemorrhage & Wound & & $1(1.8)$ & \\
& Deep & & $2(3.6)$ & \\
Wound dehiscence & Superficial & & $1(1.8)$ & \\
Anastomotic leak & & $1(1.6)$ & $5(9.1)$ & $4(2.5)$ \\
Infection & Wound & & $3(5.4)$ & \\
& Deep & $1(1.6)$ & $5(9.1)$ & $3(1.9)$ \\
& PUO & $11(18.0)$ & $11(20.0)$ & $23(14.6)$ \\
& Septicemia & & $1(1.8)$ & \\
& Chest & $3(4.9)$ & $12(21.8)$ & $7(4.4)$ \\
System failure & Urinary & $4(6.6)$ & $5(9.1)$ & $6(3.8)$ \\
& Renal & & $3(5.4)$ & $1(0.6)$ \\
& Respiratory & $1(1.6)$ & $2(3.6)$ & \\
Innominate & Cardiac & $1(1.6)$ & $3(5.4)$ & \\
Death & Hypotension & & & $1(0.6)$ \\
& & $5(8.2)$ & $3(5.4)$ & $8(5.1)$ \\
& & $1(1.6)$ & $2(3.6)$ & \\
\hline
\end{tabular}


Table 6 Rui Jin Hospital classification of complications

\begin{tabular}{|c|c|}
\hline Classification & Complications \\
\hline Minor & $\begin{array}{l}\text { Infection: superficial wound infection, deep } \\
\text { infection }^{\mathrm{a}} \text {, chest infection }{ }^{\mathrm{a}} \text {, urinary infection, } \\
\text { septicemia, and pyrexia of unknown origin }^{\mathrm{a}} \\
\text { Miscellaneous: superficial wound dehiscence, } \\
\text { wound hemorrhage, impaired renal function }{ }^{\mathrm{a}} \text {, } \\
\text { deep venous thrombosis }{ }^{\mathrm{a}} \text {, and hypotension }\end{array}$ \\
\hline Moderate & $\begin{array}{l}\text { Infection: deep infection }{ }^{\mathrm{b}} \text {, chest infection }{ }^{\mathrm{b}} \text {, and } \\
\text { pyrexia of unknown origin }{ }^{\mathrm{b}} \\
\text { Miscellaneous: deep wound dehiscence, impaired } \\
\text { renal function }^{\mathrm{b}} \text {, and deep venous thrombosis } \\
\text { Innominate }^{\mathrm{b}}\end{array}$ \\
\hline Severe & $\begin{array}{l}\text { Systemic: cardiac failure, respiratory failure, } \\
\text { pulmonary embolus, hypotension }{ }^{\mathrm{c}} \text {, and death } \\
\text { Surgical: deep hemorrhage, deep infection }{ }^{\mathrm{c}} \\
\text { anastomotic leak } \\
\text { Innominate: complications with postoperative } \\
\text { stay }>30 \text { days }\end{array}$ \\
\hline
\end{tabular}

${ }^{\text {a }}$ Postoperative $\leq 15$ days)

${ }^{\mathrm{b}}$ Postoperative $>15$ days

${ }^{\mathrm{c}}$ Requiring laparotomy

with minor, moderate, and severe complications was categorized into the severe complication group. The duration of postoperative stay (Table 7) was significantly shorter $(P=$ $0.000)$ and the rate of moderate and severe complications was significantly lower $(P=0.001)$ in the specialized unit (Table 8).

\section{Discussion}

\section{Postoperative complications}

The rate of early postoperative complications is relatively high after gastric cancer surgery [10-12]. However, in the present study, POSSUM predicted morbidity satisfactorily, which means the surgical outcome was acceptable and was comparable to that in other specialized centers around the world [10, 11, 13-15]. In addition, most of the complications were minor or moderate; and, in general, most of these complications (such as pyrexia of unknown origin) were considered to have little clinical importance.

Table 7 Duration of postoperative stay per unit

\begin{tabular}{lccc}
\hline Postoperative days & \multicolumn{4}{l}{ No. (\%) of patients per unit } \\
\cline { 2 - 4 } & A & B & C \\
\hline$\leq 15$ & $43(70.5)$ & $26(47.3)$ & $132(84.1)$ \\
$16-30$ & $14(23.0)$ & $18(32.7)$ & $20(12.7)$ \\
$>30$ & $4(6.6)$ & $11(20.0)$ & $5(3.2)$ \\
\hline
\end{tabular}

$P=0.000$
Table 8 No. (percentage) of complications per unit

\begin{tabular}{lrcr}
\hline Complication & \multicolumn{1}{l}{ A } & B & \multicolumn{1}{l}{ C } \\
\hline Null & $39(63.9)$ & $24(43.6)$ & $108(68.8)$ \\
Minor & $8(13.1)$ & $5(9.1)$ & $25(15.9)$ \\
Moderate & $11(18.0)$ & $16(29.1)$ & $18(11.5)$ \\
Severe & $3(4.9)$ & $10(18.0)$ & $6(3.8)$ \\
\hline
\end{tabular}

$P=0.001$

\section{Definition of complications in POSSUM}

There are a number of complications (e.g., pancreatic or biliary leakage, chylus leakage, ileus, enteroplegia, and pulmonary complications) that are not defined in POSSUM [8]. Although some of these complications seem to be minor, they can markedly extend the postoperative length of stay and treatment cost and should not be ignored. Therefore, POSSUM may need some modifications to be appropriate for major surgical interventions. Furthermore, for a more feasible and objective comparison, further stratification of complications according to severity was needed; we applied the RJH classification for this purpose.

\section{Interpretation of prediction}

Any system for prediction is only for reference but should be considered seriously before making any crucial decisions in surgical care. The results of a comparative audit with POSSUM cannot be used to attribute blame. Patient care is the responsibility of individual surgeons, but outcomes often depend on a large multidisciplinary team comprising surgeons, anesthetists, intensive care staff, junior doctors, and nurses. Where an audit shows a change in morbidity rates and a significant increase in the O-to-E ratio, the practice of the entire team should be reviewed [3]. Overreaction should be avoided, and the causes of deficiencies in quality should

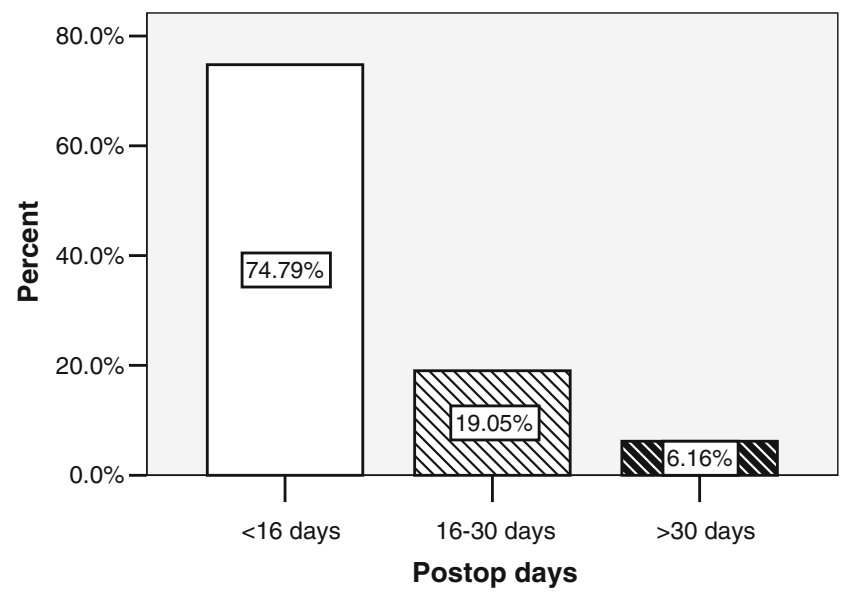

Fig. 2 Distribution chart of overall postoperative duration 
be carefully examined. Variations in outcome will occur over time; but if deteriorating O-to-E ratios can be identified early, remedial action can be taken [1].

\section{Comparison of surgical outcomes}

To ensure the safe practice of gastric cancer surgery, there must be a standard auditing system for evaluating surgical outcomes. If POSSUM is capable of risk-adjusting the case mix, perhaps the worst outcome of a unit should be attributed to the level of surgical experience, as the population characteristics, hospital resources, anesthesia, and postoperative nursing are almost the same in a given hospital.

It can be argued, however, that the POSSUM system is unable to provide risk adjustment for the case mix; in that case, comparing surgical outcomes in different units on the basis of POSSUM may be biased. The differences in predicted morbidity rates among units may be attributed to differences in population characteristics that are not adequately addressed by the 18 POSSUM variables. These include surgical disease state, comorbidities, and genetic and socioeconomic factors [16]. The differences may also be related to inadequate scoring of the magnitude of the operation; for example, all radical gastrectomies are awarded the highest score of 8 , but there may be differences in surgical extent for radical gastrectomy among different units. The extent of surgery for gastric cancer is highly heterogenous among different centers, and certainly there are differences in morbidity rates associated with different extents of surgery, even though they are all commonly denoted as radical resection [11-15, 17, 18]. Moreover, it is difficult to compare the outcome by a scoring system if there is no comparable national database available for surgical outcomes. However, the findings of this study, at the very least, demand further investigation of the potential causes of differences in surgical outcomes among different units.

\section{Risk factors for postoperative complications}

Univariate and multivariate analysis identified gender, preoperative COPD, intraoperative blood loss, and the particular unit (ward) as risk factors for early postoperative complications. However, a larger cohort of patients is necessary to support these conclusions, and if there is disparity in the complication rates between genders then further study will be focused in this direction. Given that preoperative pulmonary disease may be responsible for higher rates of postoperative pulmonary infection, further preventative treatment strategies should be considered seriously. The role of intraoperative blood loss as a risk factor for postoperative complications has been previously reported by other authors and may be related to blood transfusion, which is said to be associated with immunologic disorders [19-21]. Other factors such as age, anemia, hypoalbuminemia, and hyperglycemia, which are generally considered to be associated with higher complication rates, did not appear to be risk factors in this study. Further prospective studies are needed to support these data.

\section{Conclusion}

The preoperative variables of surgical unit, gender, concomitant COPD, and intraoperative blood loss were independent risk factors for early postoperative complications in gastric cancer surgery. However, a prospective study involving a larger cohort is necessary to confirm this result. In our experience, the rate of postoperative complications in gastric cancer surgery is relatively higher in the hands of inexperienced surgeons. We conclude that surgical experience and work volume greatly influence the outcome, which is inversely proportional to the rate of complications. Our data support the conclusion that surgery for patients with gastric cancer should be performed at specialized centers. Internal comparative audits of morbidity using a valid system for risk-adjusted evaluation of surgical outcomes such as POSSUM may help to improve overall surgical outcomes. More prospective studies are necessary to investigate the disparity in complication rate among different units.

Acknowledgement The authors thank Shanghai Institute of Digestive Surgery for funding this research, Dr Miao Qiong for her assistance in designing of data collection charts, computer engineer Mr. Dipendra Kumar Sah for designing a special POSSUM calculator and database program, the statistic bureau of Rui Jin hospital for providing records of patients, and Mr. Sun Jing Jian, officer of patient record, for his enthusiasm and support.

Open Access This article is distributed under the terms of the Creative Commons Attribution Noncommercial License which permits any noncommercial use, distribution, and reproduction in any medium, provided the original author(s) and source are credited.

\section{Appendix}

Definitions of morbidity by Copeland et al.

1. Hemorrhage: wound hemorrhage: local haematoma requiring evacuation. Deep hemorrhage: postoperative bleeding requiring re-exploration.

2. Chest infection: production of purulent sputum with positive bacteriological cultures, with or without chest radiography changes or pyrexia, or consolidation seen on chest radiograph. 
3. Wound infection: wound cellulitis or the discharge of purulent exudates.

4. Urinary infection: the presence of $>10{ }^{5}$ bacteria per milliliter with the presence of white cells in the urine, in previously clear urine.

5. Deep infection: the presence of an intra-abdominal collection confirmed clinically or radiologically.

6. Septicemia: positive blood culture.

7. Pyrexia of unknown origin: any temperature above $37^{\circ} \mathrm{C}$ for more than $24 \mathrm{~h}$ occurring after the original pyrexia following surgery (if present) had settled, for which no obvious cause could be found.

8. Wound dehiscence: superficial or deep wound breakdown.

9. Deep venous thrombosis and pulmonary embolus: when suspected, confirmed radiologically by venography or ventilation-perfusion scanning or diagnosed at postmortem.

10. Cardiac failure: symptoms or signs of left ventricular or congestive cardiac failure which required an alteration from preoperative therapeutic measures.

11. Impaired renal function: arbitrarily defined as an increase in blood urea of $>5 \mathrm{mmol} / \mathrm{l}$ from preoperative levels.

12. Hypotension: a fall in systolic blood pressure below $90 \mathrm{mmHg}$ for more than $2 \mathrm{~h}$ as determined by sphygmomanometer or arterial pressure transducer measurement.

13. Respiratory failure: respiratory difficulty requiring emergency ventilation.

14. Anastomotic leak: discharge of bowel content via the drain, wound, or abnormal orifice.

\section{References}

1. Copeland Graham Paul CHM (2002) The POSSUM system of surgical audit. Arch Surg 137(1):15-19 doi:10.1001/archsurg.137.1.15

2. Whiteley MS, Prytherch D, Higgins B et al (1996) An evaluation of the POSSUM surgical scoring system. Br J Surg 83:812-815 doi:10.1002/bjs. 1800830628

3. Neary WD, Heather BP, Earnshaw JJ (2003) The physiological and operative severity score for the enumeration of mortality and morbidity (POSSUM). Br J Surg 90:157-165 doi:10.1002/bjs.4041

4. Jones HJ, de Cossart L (1999) Risk scoring in surgical patients. Br J Surg. Feb 86(2):149-157

5. Brunelli A, Fianchini A, Al Refai M et al (2001) Internal comparative audit in a thoracic surgery unit using the physiological and operative severity score for the enumeration of mortality and morbidity (POSSUM). Eur J Cardiothorac Surg 19:924-928 doi:10.1016/S1010-7940(01)00682-0
6. Sagar PM, Hartley MN, MacFie J et al (1996) Comparison of individual surgeon's performance: risk-adjusted analysis with POSSUM scoring system. Dis Colon Rectum 39:654-658 doi:10.1007/BF02056945

7. Mohil RS, Bhatnagar D, Bahadur L et al (2004) POSSUM and PPOSSUM for risk-adjusted audit of patients undergoing emergency laparotomy. Br J Surg 91:500-503 doi:10.1002/bjs.4465

8. Copeland GP, Jones D, Walters M (1991) POSSUM: a scoring system for surgical audit. Br J Surg 78:355-360 doi:10.1002/ bjs. 1800780327

9. Wijesinghe LD, Mahmood T, Scott DJA et al (1998) Comparison of POSSUM and the Portsmouth predictor equation for predicting death following vascular surgery. Br J Surg 85:209-212 doi:10.1046/j.1365-2168.1998.00709.x

10. Viste A, Haugstvedt T, Eide GE et al (1988) Postoperative complications and mortality after surgery for gastric cancer. Ann Surg 207(1):7-13 doi:10.1097/00000658-198801000-00003

11. Lo S-S, Wu C-W, Shen K-H et al (2002) Higher morbidity and mortality after combined total gastrectomy and pancreaticosplenectomy for gastric cancer. World J Surg 26:678-682 doi:10.1007/ s00268-001-0289-8

12. Yasuda K, Shiraishi N, Adachi Y (2001) Risk factors for complications following resections of large gastric cancer. Br J Surg 88:873-877 doi:10.1046/j.0007-1323.2001.01782.x

13. Marrelli D, Pedrazzani et al (2007) Complications after extended (D2) and super extended (D3) lymphadenectomy for gastric cancer: analysis of potential risk factors. Ann Surg Oncol 14:25-33 doi:10.1245/s10434-006-9063-3

14. Danielson H, Kokkola A, Kiviluoto $T$ et al (2007) Clinical outcome after D1 vs D2-3 gastrectomy for treatment of gastric cancer. Scand J Surg 96(1):35-40

15. Yonemura Y, Wu CC, Fukushima N et al (2006) Operative morbidity and mortality after D2 and D4 extended dissection for advanced gastric cancer: a prospective randomized trial conducted by Asian surgeons. Hepatogastroenterology 53(69): 389-394

16. Bennett-Guerrero E, Hyam JA, Shaefi S et al (2003) Comparison of P-POSSUM risk-adjusted mortality rates after surgery between patients in the USA and the UK. Br J Surg 90:1593-1598 doi: $10.1002 / b j s .4347$

17. Pedrazzani C, Marrelli D, Rampone B et al (2007) Postoperative complications and functional results after subtotal gastrectomy with Billroth II reconstruction for primary gastric cancer. Dig Dis Sci 52:1757-1763 doi:10.1007/s10620-006-9655-6

18. Sano T, Sasako M, Yamamoto S et al (2004) Gastric cancer surgery: morbidity and mortality results from a prospective randomized controlled trial comparing D2 and extended paraaortic lymphadenectomy-Japan Clinical Oncology Group Study 9501. J Clin Oncol 22:2767-2773 doi:10.1200/JCO.2004.10.184

19. Arnaud A, Yves P, Danielle T et al (2002) Factors associated with clinically significant anastomotic leakage after large bowel resection: multivariate analysis of 707 patients. World J Surg 26 (4):499-502

20. Takahashi T, Hashimoto H, Hino Y et al (2000) Risk factors of anastomotic leak following operations for gastric cancer in the elderly. Nippon Ronen Igakkai Zasshi 37(4):340-343

21. Yasuda K, Shiraishi N, Adachi Y et al (2001) Risk factors for complications following resection of large gastric cancer. Br J Surg 88:873-877 doi:10.1046/j.0007-1323.2001.01782.x 